\title{
ARTICLE Systematic manipulations of the biological stress systems result in sex-specific compensatory stress responses and negative mood outcomes
}

\author{
Nida $\mathrm{Ali}^{1,2}$, Jonas P. Nitschke (iD), Cory Cooperman ${ }^{1}$, Mark W. Baldwin ${ }^{1}$ and Jens C. Pruessner ${ }^{3,4}$
}

\begin{abstract}
Women are twice as likely as men to be diagnosed with anxiety and mood disorders. One potential underlying mechanism is sex differences in physiological and psychological responses to stress; however, no studies to date have investigated this proposed mechanism experimentally. In a double-blind, placebo-controlled design, pharmacological challenges were administered to individually suppress the hypothalamic-pituitary-adrenal (HPA) axis, or the sympathetic nervous system (SNS) prior to stress exposure, to investigate sex differences in the resulting cross talk among the physiological and psychological stress responses. Sexspecific compensatory patterns and psychological effects emerged when the stress systems were manipulated. Men demonstrated heightened SNS reactivity to stress when the HPA axis was suppressed, and greater HPA reactivity after SNS suppression. This ability to react appropriately to the stressor, even with one system, did not lead to significant negative mood effects. In women, higher baseline activation (but dampened reactivity to stress) of SNS or HPA was observed when the other system was suppressed. This was coupled with worsened mood in response to stress when either stress system was compromised. Our results indicate that men and women may be differentially sensitive to fluctuations of their stress systems. This might be a potential link that underlies the sexual dimorphism in vulnerability for psychopathology.
\end{abstract}

Neuropsychopharmacology (2020) 45:1672-1680; https://doi.org/10.1038/s41386-020-0726-8

\section{INTRODUCTION}

Men and women differ in their biological and psychological responses to stress [1, 2]. This difference is proposed as one potential mechanism to explain why women are at greater risk of developing anxiety and mood disorders [3, 4]. Both anxiety and mood disorders are also associated with dysregulated biological responses to stress [5]. In this regard, while sex differences in stress reactivity are often studied separately for individual stress systems, the specific mechanisms that underlie these differences, and how they might contribute to mood dysregulations have not been examined while considering possible interactions among the stress systems. We pharmacologically manipulated the physiological stress systems to systematically investigate: (1) the interrelationships among them in the context of acute stress; (2) the consequential effects on subjective stress and mood; and (3) criticality, to compare these effects among men and women.

The biological stress response is characterized by the synchronized activity of the autonomic nervous system (ANS) and hypothalamic-pituitary-adrenal (HPA) axis. Stimulation of the ANS immediately activates the sympathetic nervous system (SNS) branch, triggering catecholamine release from the locus coeruleus, resulting in increased heart rate $(\mathrm{HR})$, blood pressure, and secretion of salivary alpha-amylase [6]. Activation of the slower acting HPA axis results in the secretion of corticotropin-releasing hormone (CRH), adrenocorticotropic hormone (ACTH), and cortisol [7]. Once activated, these systems play a role in the adaptive physiological, affective, cognitive, and behavioral responses to stress [8].

Previous studies have found that compared to women, men mount stronger HPA axis responses to psychosocial stress, resulting in greater ACTH and cortisol release $[9,10]$. Pharmacological administration studies provide further evidence for this sexual dimorphism, with women exhibiting greater pituitary sensitivity to $\mathrm{CRH}$ [11], and adrenal-cortex sensitivity to ACTH signals [12] suggesting constitutional sex differences in HPA reactivity. These disparities have been proposed to stem from differential levels of circulating gonadal hormones and corticosteroid-binding globulin (CBG) in men and women, and their interactions with cortisol [13]. Moreover, in women HPA axis responses fluctuate throughout the menstrual cycle [1], which is also associated with changing levels of gonadal hormones and CBG. With respect to SNS, men exhibit higher blood pressure and catecholamine responses to stress [9], while women have more pronounced HR reactivity [12]. Fewer studies have investigated sex differences in SAA responses, and inconsistent findings are reported, with some studies reporting increased SAA reactivity in women $[14,15]$, and others finding no significant sex differences $[16,17]$. Sex differences are also observed in affective responses to stress, with increased subjective stress, and negative mood (e.g., anger, depression, fear, anxiety) in women [15].

One potential mechanism for this might be related to sex/ gender differences in underlying social motivational factors,

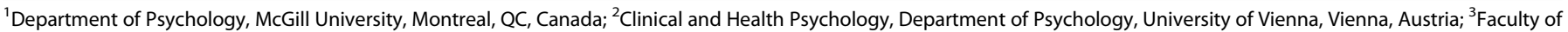
Medicine, McGill Centre for Studies in Aging, McGill University, Montreal, QC, Canada and ${ }^{4}$ Department of Psychology, University of Konstanz, Konstanz, Germany Correspondence: Nida Ali (nida.ali@mail.mcgill.ca)

Received: 7 October 2019 Revised: 14 May 2020 Accepted: 28 May 2020

Published online: 4 June 2020 
particularly during stress. Taylor et al. [18] proposed that during stress men engage in fight-or-flight responses, whereas in women, stress induced sympathetic and HPA activation modulates a tendand-befriend approach that is characterized by behavioral responses that promote the creation and maintenance of social bonds. Likewise, Tamres et al. [19] showed that men and women differ in their appraisal and coping responses to stress, and that some of these effects might be dependent on the nature of the stressor. Relatedly, Stroud et al. [2] found that men had higher stress responses to stressors that were associated with challenge and competition, whereas women demonstrated stronger physiological reactivity and psychological distress following interpersonal stressors such as social rejection. These findings suggest that while both men and women show robust SNS, HPA, and psychological responses to stress, the magnitude of these responses appear to be driven in part by sex/gender specific effects.

While sex differences in stress reactivity within individual stress systems are frequently studied, a systematic investigation, through experimental manipulation, of the interactions between stress systems in men and women is missing. Histological evidence has identified reciprocal neural connections between, for example, the hypothalamus and locus coeruleus [7], suggesting an important mechanism of cross talk and interaction between the two systems. Consequently, studies have begun examining this cross talk during acute stress. Using a cross-correlational approach, Engert et al. [20] demonstrated that stress induced sAA increase correlates with cortisol in a time-lagged manner, with sAA preceding cortisol by around 14 minutes. Recently our group has utilized pharmacological challenges to experimentally investigate the interrelationships between the stress systems, and found that suppressing the HPA axis resulted in compensatory increases in $\mathrm{HR}$ and subjective-stress responses to stress. Likewise, following SNS suppression, cortisol reactivity was heightened, indicating that the stress systems do indeed cross talk [21, 22]. While these studies demonstrated the efficacy of using pharmacological manipulations to examine interactions among the stress systems, they did not address possible sex differences (all participants were male), or psychological outcomes such as changes in mood.

Here, we investigated sex differences in the interrelationships between physiological and psychological responses to acute stress. While previous research has found important associations between chronic dysregulations of the stress systems and anxiety and mood disorders (for a review see [23]), here we investigated whether an acute-one time-pharmacological dysregulation of individual stress systems would result in altered stress responses, and negative psychological effects in men and women. Thus, in a double-blind placebo (PLC)-controlled design, we administered dexamethasone (DEX) or propranolol (PROP) before stress induction. DEX is a synthetic glucocorticoid that binds to peripheral and pituitary glucocorticoid receptors, and suppresses ACTH and cortisol production [24]. PROP is a $\beta 1$ and $\beta 2$ receptor antagonist that has been shown to suppress SNS activity [25]. The following hypotheses were tested: (1) under PLC, HPA axis stress reactivity would be higher in men than women, who, in turn, would show increased SNS (specifically SAA and HR), and psychological responses [1]; (2) following HPA suppression (DEX administration), SNS responses would be higher than PLC. However, since women routinely have lower cortisol responses, compensatory SAA and HR responses would be higher in women than men [21]; (3) following SNS suppression (PROP administration), men would compensate with higher cortisol, compared with PLC, and women [22]; (4) since dysregulations of the stress systems are linked to negative affect $[26,27]$, manipulating the systems should result in increased subjective stress and negative mood in men and women. While these effects are typically more pronounced in women [28], a previous study by our group did not find sex differences in mood dysregulations following stress [29].
We therefore explored whether suppressing either stress system would result in differential effects on subjective stress and mood in women and men.

\section{MATERIALS AND METHODS}

Participants (18-35 years old) were recruited via advertisements posted on the McGill University website classifieds section and screened for eligibility. Exclusion criteria included recreational drug use, consuming $>10$ alcoholic beverages/week, smoking $>7$ cigarettes/day, current or previous significant medical or psychiatric illnesses, or use of medications that affect HPA functioning. Women were tested during the luteal phase of their menstrual cycle (17-28 days after menstruation onset). To establish luteal phase, women recorded the first day of their menses, for two consecutive cycles before testing. Participants provided informed consent for the study, which was approved by the McGill University Faculty of Medicine Institutional Review Board.

Sample size was determined via power calculations based on previous pilot studies in our lab [21, 22] using the same paradigm. In these studies, the difference between groups of 15 subjects (per cell) yielded a medium to high effect size of $f=0.25-0.4$ [30]. For the current study, using the statistical software G*Power [31], an assumed effect size of 0.30 , power $\geq 0.95$, and $a=0.05$, we estimated that a cell size of at least 16 participants would be needed to detect an effect. A total of 60 men (mean age $=23.28$, range $=18-35, S D \pm 3.85$ ), and 61 women (mean age $=23$ range $=$ $18-35, S D \pm 4.47)$ were randomly assigned to receive: PLC (20 men, 21 women), DEX (20 men, 20 women), or PROP ( 20 men, 20 women). Participants took $2 \mathrm{mg}$ of DEX (or PLC) at bedtime the night before stress induction, and $80 \mathrm{mg}$ of PROP (or PLC) $1 \mathrm{~h}$ before stress. A physician was on call each day in case of serious side effects. No adverse reactions occurred in any participants. To ensure double blinding every subject received two pills, one the night before, and one $60 \mathrm{~min}$ before stress, resulting in the following experimental conditions: double PLC, DEX-PLC, and PLC-PROP.

\section{Testing paradigm}

Testing occurred on 2 consecutive days in our laboratory. On day 1, participants received one pill (PLC or DEX) with instructions to take it at bedtime. The next morning, on day 2 of testing, participants received one pill (PLC or PROP) 1 hour before the onset of stress, and were then seated in a waiting room for a 60min rest period for the PROP to take effect. Participants were then exposed to the psychosocial stressor (described below), following which they completed questionnaires to assess stress-related changes in subjective stress and mood. More detailed information about the testing protocol is described in [29]. A design figure of the day 2 protocol is presented in the Supplementary Materials (Fig. S3).

\section{Stress paradigm}

Psychosocial stress was induced via the Trier Social Stress Test (TSST; [32]). The TSST consists of a 10-min anticipation phase, followed by a testing phase encompassing a 5 -min speech and a 5 -min mental-arithmetic task, performed in front of trained confederates. The TSST reliably produces increases in cortisol, sAA, HR, subjective stress, and negative mood $[6,26,32]$.

\section{Stress biomarkers}

Cortisol and SAA were analyzed from saliva samples (Sarstedt Inc., Nümbrecht, Germany). Cortisol (nmol/l) was measured using a timeresolved fluorescence immunoassay [33]. Alpha-amylase (U/ml) was determined using the enzyme kinetic method [20]. HR was measured using an ambulatory sphygmomanometer (A\&D Company, Tokyo, Japan). Assessments were made at nine timepoints, in 10-min intervals, throughout the experiment (Baseline: $-20,-10$, stress: 0 , post stress: $+10,+20,+30,+40,+50,+60 \mathrm{~min})$. 


\section{Psychological assessment}

Participants provided self-reports of subjective stress and mood. Subjective stress was measured using a ten-point visual analog scale [(VAS; [34]) asking "How stressed do you feel right now?" Responses ranged from "not at all" to "extremely." The VAS was administered with saliva samples at timepoints $-10,0,+10,+20$. The Profile of Mood States (POMS; [35]) questionnaire was administered at baseline (before stress); during the anticipation period before TSST; and immediately after the TSST. The 66 items of the POMS are used to compute six subscales and a composite measure of total mood disturbance (TMD). Based on our previous work [29], and given the stress-related context of this study, we focused exclusively on the "depression," "anxiety," and "anger" subscales, and TMD.

\section{Statistical analyses}

One participant (male in the DEX condition) was excluded due to missing mood measures. Three participants were excluded on account of unsuccessful pharmacological suppression (biomarker values $>3$ std-dev. above mean) via DEX (one female), and PROP (one male, one female). Final analyses were conducted with 117 participants (PLC: $n=41$; DEX: $n=38$; PROP: $n=38$ ).

Two-way (sex $\times$ drug) MANOVAs were conducted for age, depressive symptoms, body mass index, and self-esteem as dependent variables, to ensure that the groups did not differ on these variables.

Biomarkers. Mixed-effects multilevel models (MELM; [36]) were computed to examine the relationship between sex, and repeated measures of biomarkers (cortisol, sAA, HR), in the PLC condition. Sex $($ male $=0$, female $=1$ ), sampling times (time $1=0$ ), and sex $\times$ time interaction were entered as fixed effects. Based on the recommendation by Barr et al. [37], the intercept of time nested within subject was added as a random effect.

Separate MELMs were computed to examine the relationships between sex, drug, and repeated measures of biomarkers. Sex (male $=0$, female $=1)$, drug $(P L C=0, D E X=1 ; P L C=0, P R O P=$ $1)$, sampling times (time $1=0$ ), and interactions of sex $\times$ drug; sex $\times$ time; drug $\times$ time; and sex $\times$ drug $\times$ time were entered as fixed effects. Time nested within subject was added as a random effect. Likelihood ratio tests were conducted to compare interaction models.

For all MELMs, main effects models were conducted first. If either the double-interaction model, or the triple-interaction model was deemed to be more parsimonious, as defined by a significant reduction in AIC, the most significant model was adopted. For ease of reading, here we report only the results of the best performing model. Model fit indexes comparing the different models are presented in the Supplementary Materials (Tables ST1-ST3).

Psychological outcomes. To examine the effects of drug suppression on psychological responses to stress, area under the curve increases (AUCis) for VAS, and subscales of POMS were computed [38]. Multiple imputation analyses were computed for missing mood data using the MICE [39] package to estimate missing values $(6 \%$ missing). A MANOVA was conducted to investigate the effect of stress on psychological responses in the PLC condition. AUCis for VAS, and mood were entered as dependent variables, and sex as independent variable. To investigate the effects of drug on psychological responses, we conducted MANOVAs (sex $\times$ drug), for AUCis of VAS and mood as dependent variables, and sex and drug conditions (PLC, DEX); (PLC, PROP) as independent variables.

All analyses were conducted using R, version 3.4.4 [40]. Mixedeffects analyses were conducted using the LME4 [41] package. Significant effects were decomposed using the formula by Preacher et al. [42].

\section{RESULTS}

MANOVAs revealed no significant differences in age, depressive symptoms, body mass index, and self-esteem ( $F s<0.84, p s>0.36)$, indicating that men and women across all drug groups were comparable on these factors.

\section{Manipulation check}

Based on the $1.5 \mathrm{nmol} / \mathrm{l}$ threshold for increase in cortisol from baseline to peak levels, proposed by Miller et al. [43], across all participants in the PLC condition, the TSST induced a $74.4 \%$ increase in cortisol. While, to our knowledge, no such criteria have been proposed for $S A A$, in our study, participants in the PLC condition showed a $126.6 \%$ increase in $\mathrm{SAA}$, from baseline to stress, indicating that the TSST induced significant increases in stress markers.

\section{Biomarkers}

Figure S1 in the Supplementary Materials illustrates the stress response in the PLC, DEX, and PROP conditions, separately for men and women, using untransformed values for cortisol, sAA, and HR.

Placebo. In the PLC condition, the MELM showed significant sex $\times$ time interactions for cortisol, $F(8,212.08)=3.98, p<0.001$, and sAA, $F(8,326.49)=2.77, p<0.01$. Post-hoc tests revealed that, as predicted, men had stronger cortisol responses to stress than women, 20-30 min after the TSST (timepoints 6, 7; ts > 2.37; $p \mathrm{~s}<$ 0.01 ), and women had significantly higher SAA than men at the onset of the TSST (timepoint $3 ; t=3.93, p<0.001$ ). There was a significant main effect of time on $\mathrm{HR}, F(8,216.17)=11.66, p<$ 0.001 , indicating increased HR responses to stress; however, there was no main effect of sex, nor a sex $\times$ time interaction (Fig. 1a).

Dexamethasone-placebo. Comparing DEX to PLC, the MELM revealed a significant triple interaction of sex $\times$ drug $\times$ time for cortisol, $F(8,409.85)=3.31, p \leq 0.01$. Post-hoc tests showed that cortisol levels were significantly lower across all timepoints for men and women in the DEX group, ( $p s<0.001$ ) indicating that DEX administration effectively suppressed the HPA axis (Fig. 1b).

For the intact biomarkers, we observed significant sex $\times$ time, $F(8,423.11)=2.36, p=0.01$, and drug $\times$ time, $F(8,423.11)=1.90$, $p>0.05$, interactions for $\mathrm{sAA}$, and a significant triple interaction of sex $\times$ drug $\times$ time, $F(8,432.89)=2.02, p=0.04$ for $\mathrm{HR}$.

Within-sex contrasts. Comparing between drug conditions, men in the DEX condition had marginally higher $S A A$, immediately after the TSST (timepoint $4 ; t=1.71, p=0.08$ ), and significantly greater $\mathrm{HR}$ reactivity (timepoint $4 ; t=2.19, p=0.02$ ) than PLC. This finding is consistent with previously reported effects of compensatory stress responses in men [21]. In women, the DEX group already had overall higher SAA at baseline (timepoint $2 ; t=2.05, p=0.04$ ), stress reactivity (timepoint $4 ; t=3.06, p=0.002$ ), and also recovery, $30 \mathrm{~min}$ after the TSST (timepoint 7; $t=2.33, p=0.02$ ). DEX was also associated with higher HR at baseline (timepoint 2; $t=2.41, p=0.01$ ), and 40 min post stress (timepoint $8 ; t=2.08$, $p=0.03$ ), but not reactivity (timepoint $3 ; t=1.01, p=0.3$ ), suggesting that women too showed compensatory responses, however, with a somewhat different pattern (Fig. 2).

Between-sex contrasts. Compared to men, women in DEX had higher sAA over all timepoints (all $p s<0.01$ ), and higher HR at baseline (timepoint $2 ; t=2.16, p=0.03$ ), and 50 min post stress (timepoint $8 ; t=2.22, p=0.02$ ). There was no significant sex difference in peak HR stress reactivity (timepoint $4 ; t=-0.51, p=$ 1.38) (Fig. 1b).

Propranolol-placebo. Comparing PROP to PLC, the MELM revealed a significant triple interaction of sex $\times$ drug $\times$ time for sAA $F(8,418.56)=2.61, p<0.01$, and significant drug $\times$ time interaction for $\operatorname{HR} F(8,436.49)=5.82, p<0.001$. Post-hoc tests 

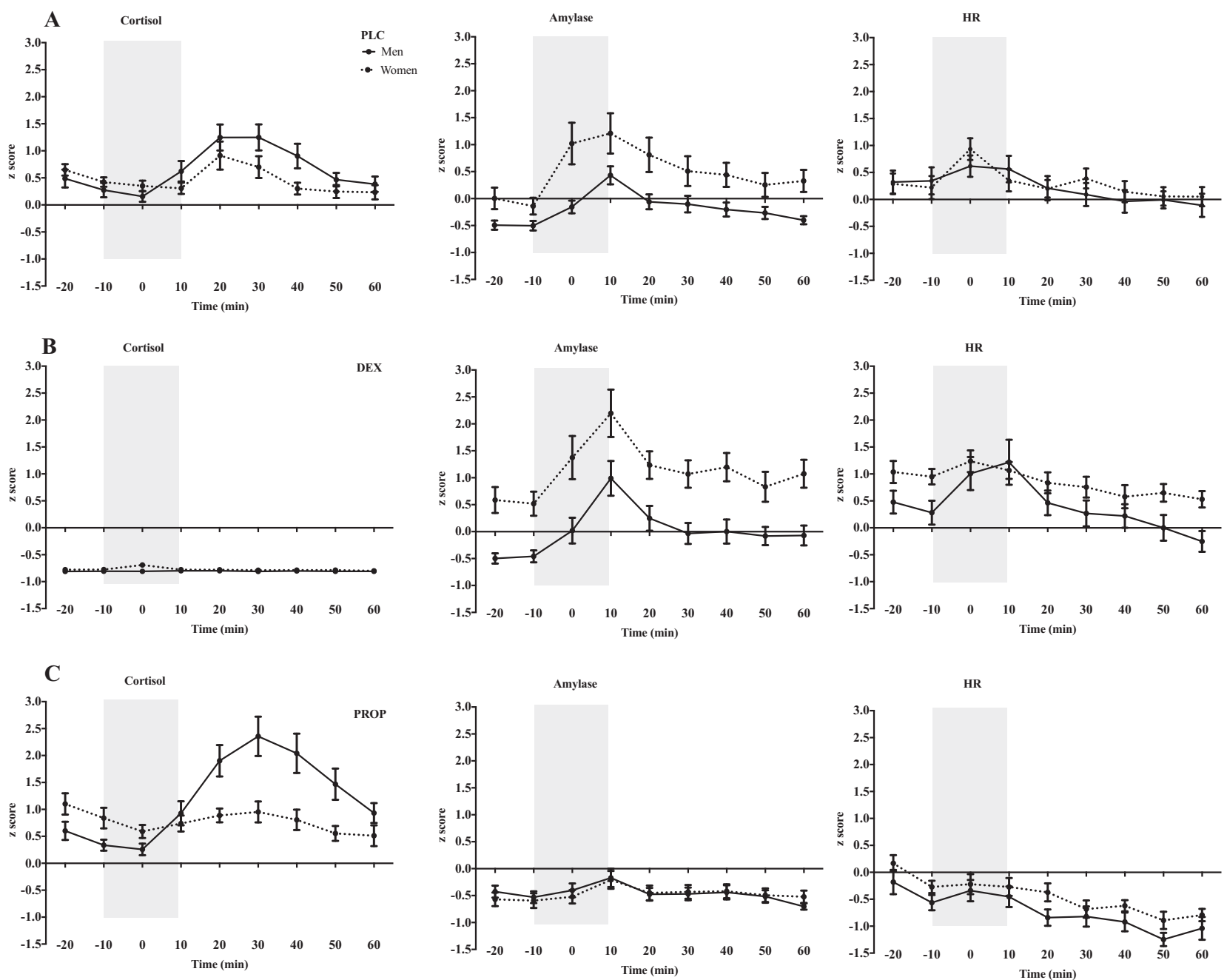

Fig. 1 Physiological stress responses in each drug condition. Z-scores of the effects of the TSST on the different stress markers in men and women for each drug condition: a placebo (PLC), b dexamethasone (DEX), and c propranolol (PROP). From left to right, cortisol, salivary alphaamylase, and heart rate. The gray bar represents the stress period that encompasses the anticipation period $(-10$ to 0$)$, and the TSST $(0$ to +10$)$.

revealed that SAA and HR were significantly lower in PROP, indicating that PROP administration effectively suppressed SNS responses ( $p s<0.01$ ) (Fig. 1c).

For cortisol we observed a significant triple interaction of sex $x$ drug $\times$ time, $F(8,385.59)=2.97, p<0.01$.

Within-sex contrasts. Comparing between drug conditions, men in the PROP group had higher cortisol at timepoints corresponding with stress response, i.e., $20-40 \mathrm{~min}$ post TSST (timepoints 5-7; ts $>2.73$; $p s<0.001$ ), replicating previous findings on compensatory HPA activity following SNS suppression in men [22]. In women, the PROP group had higher cortisol at baseline (timepoint $1 ; t=1.99, p=0.04$ ), and recovery from stress (timepoint $7 ; t=$ $1.81, p=0.07)$, but not at peak stress reactivity, i.e., $20-30 \mathrm{~min}$ post TSST, (timepoint 5,$6 ; t s<0.98 ; p s>0.32$ ); paralleling compensatory responses in men but with a somewhat different pattern (Fig. 2).

Between-sex contrasts. Women in PROP had higher cortisol at baseline (timepoints $1,2, t s>2.14$, ps $<0.03$ ) than men; however, stress response was higher in men $20-40 \mathrm{~min}$ post TSST (timepoints 5-7; ts $>4.17$, ps $<0.001$ ) (Fig. 1c).

\section{Psychological outcomes}

Across all drug conditions subjective stress and mood responses were strongly correlated with each other ( $r s>0.15, p s<0.001)$.
A MANOVA was conducted to examine whether men and women differed at baseline affective markers. The results revealed no significant effects of sex at baseline for TMD, anxiety, depression, anger, or VAS ( $F s<2.5, p s>0.11$ ).

Figure S2a, b in the Supplementary Materials illustrate the linear trajectories of POMS and subjective stress in the PLC, DEX, and PROP conditions, separately for men and women.

Placebo. In the PLC condition, the MANOVA revealed a significant main effect of sex for AUCis of depression, $F(1,39)=$ $5.51, p=0.02, \eta^{2}=0.12$, and marginal effect for anxiety, $F(1,39)=$ 3.86, $p=0.056$. Examination of means revealed that stress induction resulted in greater negative affect in women than in men (Fig. 3a). There were no sex differences on TMD, anger, or VAS ( $p s>0.19$ ). See Table 1 for mean AUCs of all psychological variables for men and women in each drug condition.

Dexamethasone-placebo. The MANOVA revealed significant sex $\times$ drug interactions for TMD, $F(1,75)=4.02, p=0.04, \eta^{2}=0.05$, anger, $F(1,75)=22.83, p<0.001, \eta^{2}=0.21$, and VAS, $F(1,75)=$ 3.67, $p \leq 0.05, \eta^{2}=0.04$, and significant main effects of sex for depression, $F(1,75)=17.91, p<0.001, \eta^{2}=0.19$, and anxiety, $F(1,75)=9.17, p=0.003, n^{2}=0.11$. 

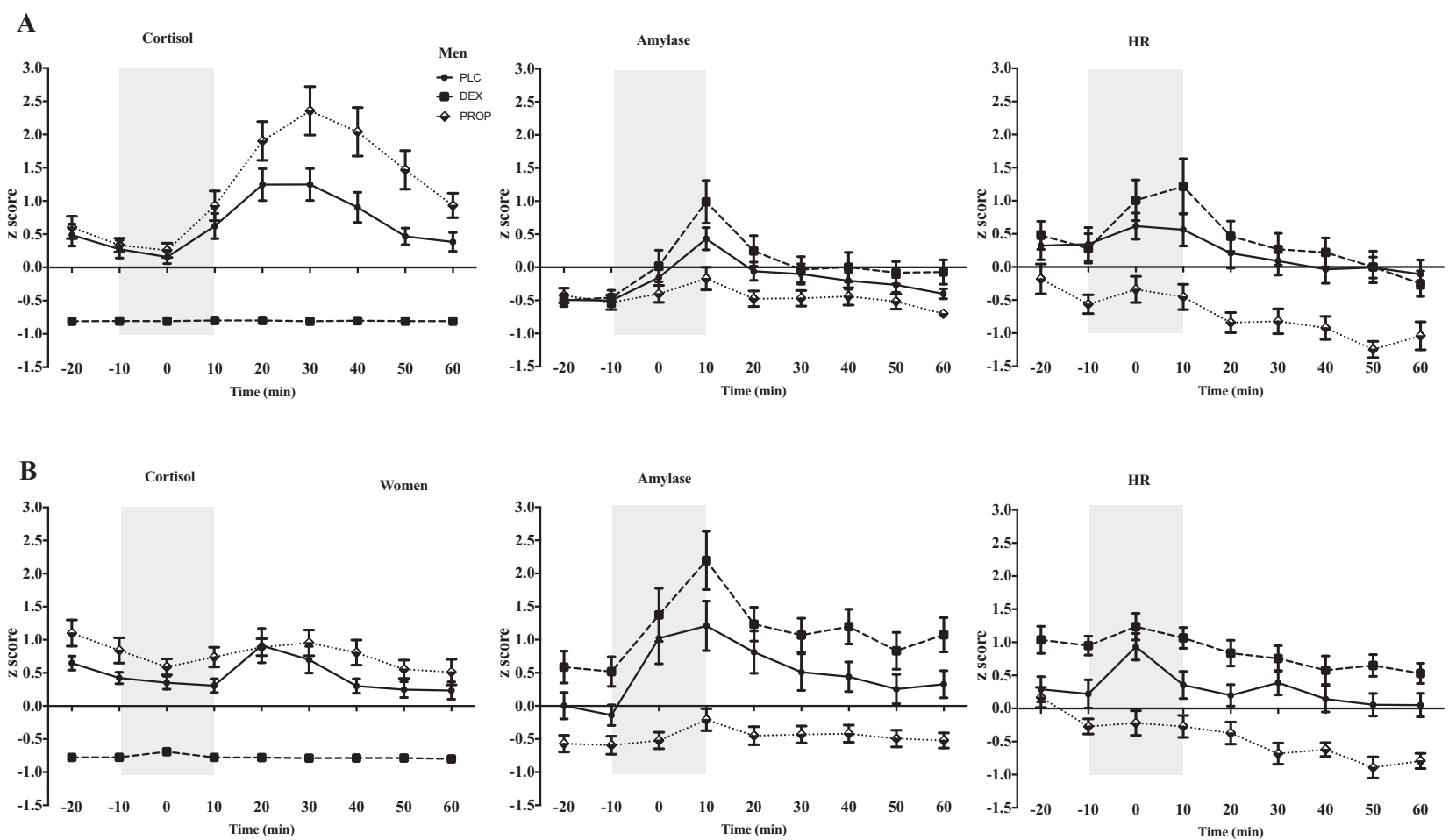

Fig. 2 Physiological stress responses in men and women. Z-scores of the effects of the TSST on the different stress markers in the placebo (PLC), dexamethasone (DEX), and propranolol (PROP) conditions, in a men, and $\mathbf{b}$ women. From left to right, cortisol, salivary alpha-amylase, and heart rate. The gray bar represents the stress period that encompasses the anticipation period $(-10$ to 0$)$, and the TSST (0 to +10$)$.

Within-sex contrasts. Comparing between drug conditions, in men the MANOVA revealed a significant main effect of anger, $F(1,37)=$ $7.40, p=0.01, \eta^{2}=0.17$. Post-hoc tests revealed that men in the DEX condition had reduced anger following stress (Fig. 4a). There were no differences between drug conditions on TMD, anxiety, depression, or VAS ( $p s>0.24)$. In women, there was a significant increase in $\mathrm{TMD}, F(1,38)=4.73, p=0.03, \eta^{2}=0.11$; anger, $F(1,38)=16.09, p<$ $0.001, \eta^{2}=0.29 ;$ and VAS, $F(1,38)=6.48, p=0.01, \eta^{2}=0.15$, indicating that women in the DEX group experienced greater subjective stress, and negative mood following stress (Fig. 4b).

Between-sex contrasts. The MANOVA revealed significant sex differences on all psychological markers: TMD, $F(1,36)=8.56, p<$ $0.01, \eta^{2}=0.19$; anxiety, $F(1,36)=5.35, p=0.03, \eta^{2}=0.13$; depression, $F(1,36)=17.95, p<0.001, \eta^{2}=0.33$; anger, $F(1,36)=27.85$, $p<0.001, \eta^{2}=0.44$; and VAS, $F(1,36)=14.21, p<0.001, \eta^{2}=0.28$, indicating that women in the DEX condition had increased subjective stress and broad mood disturbance in response to stress, compared to men (Fig. 3b).

Propranolol-placebo. The MANOVA revealed a significant sex $x$ drug interaction for anger, $F(1,75)=7.21, p<0.01, \eta^{2}=0.09$, and a main effect of sex for depression, $F(1,75)=8.64, p<0.01, \eta^{2}=$ 0.10 .

Within-sex contrasts. Comparing between drug conditions, the MANOVAs revealed no effects of drug on TMD, anxiety, depression, anger, or VAS (all $p s>0.21$ ) in men. In women, there was a marginal increase in TMD, $F(1,38)=3.30, p=0.07$, and a significant increase in anger, $F(1,38)=7.57, p<0.01, \eta^{2}=0.17$, indicating that women in the PROP group experienced greater mood dysregulation in response to stress (Fig. 4b).

Between-sex contrasts. The MANOVA revealed significant sex differences in anger, $F(1,36)=5.52, p=0.02, \eta^{2}=0.13$; and marginal differences in TMD, $(p=0.07)$, and depression, $(p=$
0.08), indicating that compared to men, women had greater mood disturbance, particularly anger, in response to stress (Fig. 3c).

\section{DISCUSSION}

In this study we individually suppressed the SNS, or the HPA axis to investigate sex differences in the cross talk between physiological and psychological responses to acute stress. Consistent with our hypotheses, when both systems were intact, men mounted a stronger HPA axis response, whereas women showed stronger SNS reactivity to stress. When either stress system was suppressed, men showed increased stress reactivity in the intact system, whereas women compensated with overall heightened biomarker levels. This differential compensatory response pattern corresponded with increased subjective stress and negative mood in women only, highlighting a potential mechanism underlying sex differences in vulnerabilities to mood and anxiety disorders.

Previous studies have shown that men typically mount stronger reactive cortisol responses to stress than women $[1,9,12]$. Our results are consistent with these findings and demonstrate that under PLC men are indeed more reliant on the HPA axis during stress. In the DEX condition, replicating previous findings [21], men compensated with increased $S A A$ and HR reactivity. Likewise, in the PROP condition, men showed increased cortisol stress reactivity, as previously shown [22]. Consistent with the literature, our results thus indicate that when either stress system is functionally compromised in men, stress reactivity of the intact system-SNS or HPA axis-is enhanced. Importantly, within men a dysregulation of either system was not associated with changes in subjective stress or mood.

Under PLC women mounted stronger SAA stress responses than men. When either stress system was suppressed, women too demonstrated compensatory responses in the intact stress 

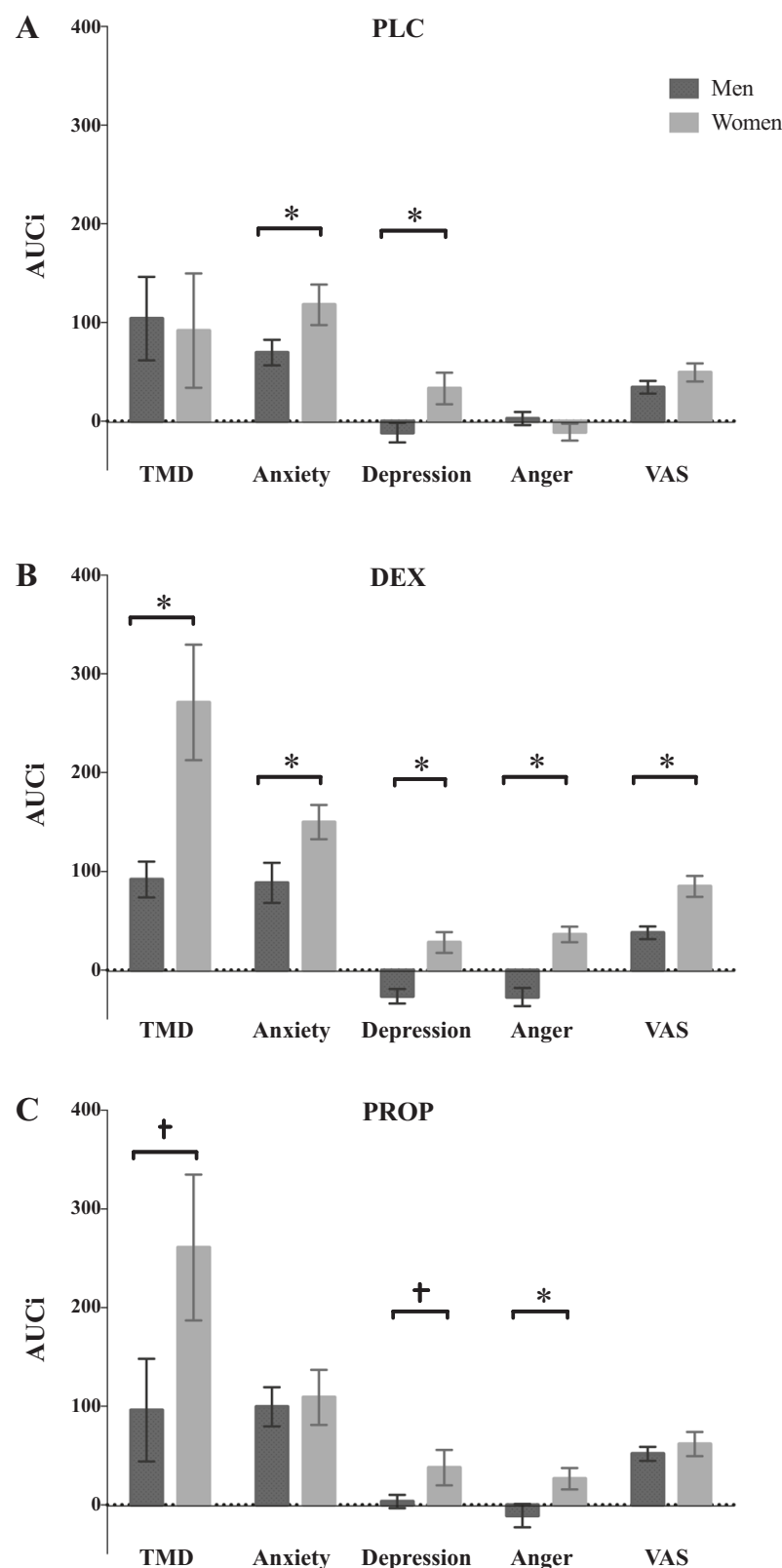

Fig. 3 Effect of TSST on mood and subjective stress. Area under the curve increase (AUCi) values (+SEM) for mood on total mood disturbance (TMD), Anxiety, depression, anger, and subjective stress (VAS) between men and women in the a placebo (PLC), b dexamethasone (DEX), and c propranolol (PROP) conditions.

systems. However in contrast to men, who had greater biomarker reactivity, women showed predominantly increased overall stress markers in the intact systems, indicating overall sensitivity of the stress pathways. Specifically, when the HPA axis was compromised, women had elevated sAA and HR, even before stress induction. Likewise, when the SNS was suppressed, compared with PLC, women had elevated cortisol, at baseline and after stress. Importantly there were no differences between DEX and PLC women at peak HR reactivity, and PROP and PLC women at peak cortisol reactivity. We interpret this to suggest that higher baseline HR and cortisol levels in response to the pharmacological manipulation prior to stress exposure may have caused a "ceiling effect," which prevented women from mounting strong reactive responses when confronted with stress. These elevated basal HR and cortisol levels may have thus potentially strained the stress system (and flattened the stress response) and prevented it from adaptively reacting to acute stress $[12,44]$.

One potential mechanism underlying this dimorphic pattern of results might be related to sex differences in gonadal hormones, specifically estrogen and progesterone. These hormones have been found to modulate the HPA axis [45, 46], and higher sensitivity to endogenous fluctuations of gonadal hormones has been associated with mood dysregulations and depression in women [47]. Importantly, since the levels of these hormones vary significantly across the life span [3] (i.e., puberty, menstrual cycle, pregnancy, menopause), women may have developed a greater reliance on the SNS to respond to and cope with stress. In addition, there is some evidence that compared with men, women objectively and subjectively experience stressful life events more frequently [13], and appear to be more vulnerable to adverse effects of stress [48]. Thus, it could be that women have adapted to continually compensate for fluctuating levels of stress hormones (in response to biological cycles, and external stressors), resulting in increased sensitivity of their stress systems to any external and internal perturbations. Additional support comes from animal studies showing that female rats are slower to adapt to repeated or chronic stressors, and in fact show higher baseline and stress reactive corticosterone levels during chronic stress inductions [49]. Likewise, in humans, increased basal and reactive cortisol levels are observed earlier in preadolescent girls, than boys [50]. Pharmacological challenges have also found that women show greater HPA axis responsivity, and decreased feedback sensitivity, compared with men [13]. Overall, this literature suggests that the stress system is highly sensitive in women, not only during stress, but also basally. Our results are consistent with this, and demonstrate that functional impairments of either stress system result in increased activity in the intact system, even before stress has occurred. Moreover, these manipulations result in significantly worse mood in women during stress. We propose that this systemic sensitivity of the stress systems impacts how women physiologically react to acutely stressful situations, and the inability to respond effectively to stress might leave them more psychologically reactive.

Psychologically, under PLC women reported greater anxious and depressed affect following stress, than men. Dysregulation of either system was further associated with increased subjective stress and negative affect in women. Previous studies have linked chronic dysregulations of the stress systems with negative psychological consequences, i.e., increased subjective stress $[21,22]$, mood disturbance [26], and higher risk for psychopathology [5]. Our results in women are consistent with these findings. One potential mechanism for this sex difference may be that women in either pharmacological condition had elevated stress biomarkers, even before stress occurred. This can be considered in the context of emotion theories that posit that the perceived stressfulness of a situation together with physiological cues of arousal impact how we assess our ability to cope with the situation. This results in feelings of competence, or a depressive state, which comprise the emotional stress response [51]. In our study, baseline activations of the stress systems may have impacted how women attended to the contextual, and/or internal bodily cues associated with stress. In addition, the inability to mount strong physiological reactive responses may have resulted in the detrimental mood outcomes we observed. Support for this comes from the literature on the mood-buffering effects of cortisol. Here, both pharmacological and psychosocial laboratory based acute stress studies have shown an inverse relationship between cortisol and negative mood, such that higher cortisol reactivity to stress has been associated with lower negative affect $[52,53]$. Moreover, a recent study by our group also exhibited this 
Table 1. Means of change in mood and subjective stress for men and women in each drug condition.

\begin{tabular}{|c|c|c|c|c|c|c|}
\hline & \multicolumn{2}{|l|}{ PLC } & \multicolumn{2}{|l|}{ DEX } & \multicolumn{2}{|l|}{ PROP } \\
\hline TMD & 104 (189) & $91.8(265)$ & 91.9 (79.4) & $271(255)$ & 96.1 (227) & 261 (323) \\
\hline Anxiety & $69.5(58.3)$ & $118(94.1)$ & $88.5(88.4)$ & $150(75.1)$ & 99.5 (86.5) & 109 (122) \\
\hline Depression & $-11.6(44.4)$ & $33.3(73.5)$ & $-26.3(32.1)$ & $28.1(45.8)$ & $3.46(29.3)$ & $37.8(78.3$ \\
\hline Anger & $2.72(29.5)$ & $-11.1(39.5)$ & $-27.8(40.1)$ & 36.2 (34.6) & $-10.9(51.2)$ & $26.5(46.9$ \\
\hline
\end{tabular}

Mean and standard deviations (in parentheses) for AUCs of mood and subjective-stress measures across drug conditions in men and women.

PLC placebo condition (no suppression), DEX dexamethasone (HPA suppression), PROP propranolol (SNS suppression), TMD total mood disturbance, VAS visual analog scale.

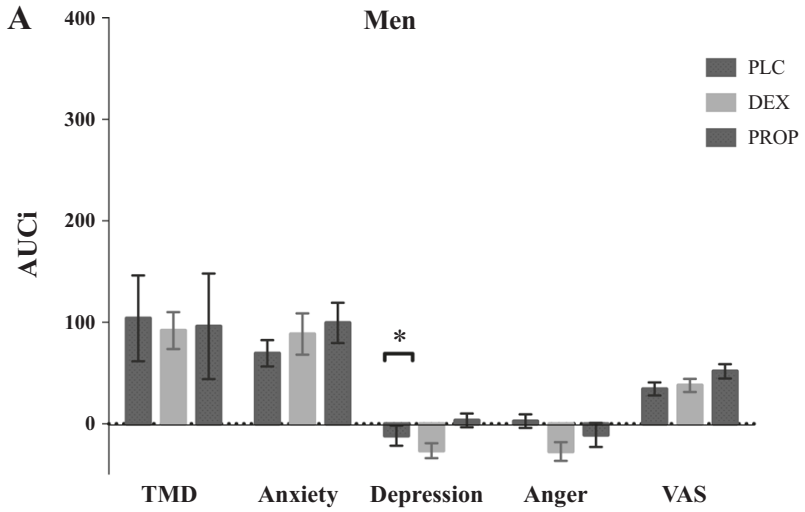

Women

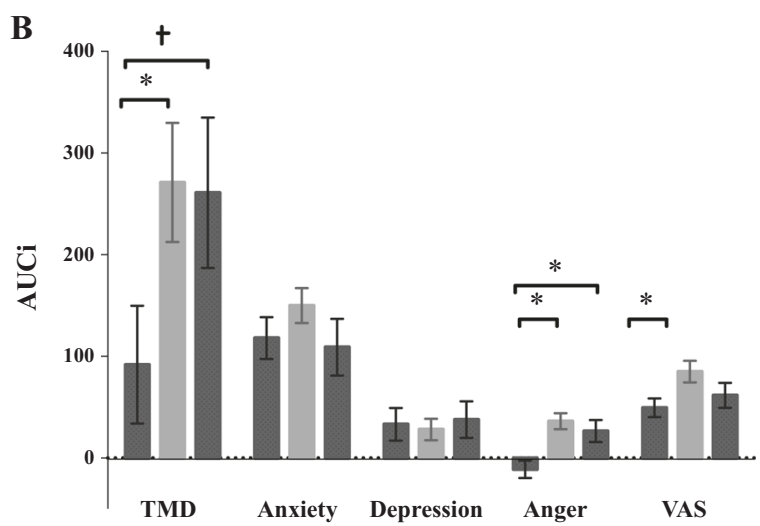

Fig. 4 Effect of TSST on mood and subjective stress. a Area under the curve increase (AUCi) values (+SEM) for mood on total mood disturbance (TMD), anxiety, depression, anger, and subjective stress (VAS) in men across all drug conditions. b Area under the curve increase (AUCi) values (+SEM) for mood on total mood disturbance (TMD), anxiety, depression, anger, and subjective stress (VAS) in women across all drug conditions.

inverse relationship such that pharmacological suppression of both stress systems resulted in increased subjective stress and negative mood [29]. However, we did not observe sex differences in that study. We suspect that this might be due to the lower statistical power, or a stronger global effect from the double suppression, which might have masked sex differences. Finally, it is important to note that while we link pharmacological suppressions of the stress systems with negative psychological consequences following laboratory stress in a healthy sample, similar adverse psychological consequences of stress system dysregulations have been observed in clinical populations [54], and individuals with severe allostatic load (e.g., histories of early life adversity and burnout) [55].

\section{LIMITATIONS AND CONCLUSION}

This study has some limitations. Firstly, while DEX was administered to suppress the HPA axis, this may not have resulted in a complete suppression during acute stress. DEX principally acts on the pituitary to suppress ACTH and cortisol production [24]. Thus given the blunted cortisol levels in the DEX condition, we can be certain that the observed outcomes were not due to peripheral increases in cortisol during stress. However since DEX does not cross the blood-brain barrier [24], acute stimulation of the HPA axis may have resulted in central increases in CRF, contributing to some of our effects. In addition, we administered PROP to effectively block SAA release by inhibiting beta-adrenergic receptor activation [22]. However, since PROP crosses the blood-brain barrier, it may have only partially blocked the effects of peripheral or central adrenaline and noradrenaline, via effects on the beta-adrenergic receptors but not via alpha adrenergic receptors [56]. Thus, we recognize that the effects of PROP and DEX on the physiology of the stress systems may be more widespread and nuanced, and that both pharmacological agents may have effects that extend beyond the observed decreases in SAA and cortisol responses. Secondly, we assessed sex as a binary factor. Given that estrogen and progesterone exert strong modulating effects on the HPA axis [13], future investigations should include assessements of these gonadal hormones to examine their role in the sex-related effects we observed. Relatedly, since we only included women in the luteal phase our results may not generalize to women in other menstrual phases, or those on oral contraceptives [57]. Moreover, given the limited age range of our participants, whether our results can be generalized across all ages is yet to be determined. In addition, given that higher biomarker reactivity to stress in men was not associated with detrimental affective responses to stress, our findings cannot comment on the potential mechanisms that underlie the onset and maintenance of stress-related neuropsychiatric disorders in men. Relatedly, we used limited measures to assess psychological effects of stress. Future studies should consider investigating affective and cognitive processes (e.g., social threat assessment, emotional memory), since these may have been altered in men (and women) across drug conditions.

Notwithstanding these limitations, this study demonstrates that men and women differentially rely on the HPA axis and SNS during stress. Importantly, sex-specific compensatory patterns and affective outcomes emerge when either stress system is functionally compromised, highlighting a potential mechanism underlying increased vulnerability for psychopathologies in women. The authors declare no competing interests. 


\section{AUTHOR CONTRIBUTIONS}

NA and JCP conceived and designed the study. NA and CC collected the data. NA and JPN conducted statistical analyses. NA, JPN, MWB, and JCP interpreted the findings. $\mathrm{NA}, \mathrm{MWB}$, and JCP wrote and edited the manuscript. CC and JPN reviewed and edited the manuscript.

\section{FUNDING AND DISCLOSURE}

This study was supported by research grants awarded by the Canadian Institutes of Health Research to JCP (grants no. 125881 and 148728). NA was awarded the doctoral research award by the Fonds de Recherche du Québec-Santé.

\section{ADDITIONAL INFORMATION}

Supplementary Information accompanies this paper at (https://doi.org/10.1038/ s41386-020-0726-8)

Publisher's note Springer Nature remains neutral with regard to jurisdictional claims in published maps and institutional affiliations.

\section{REFERENCES}

1. Kirschbaum C, Kudielka BM, Gaab J, Schommer NC, Hellhammer DH. Impact of gender, menstrual cycle phase, and oral contraceptives on the activity of the hypothalamus-pituitary-adrenal axis. Psychosom Med. 1999;61:154-62.

2. Stroud LR, Salovey P, Epel ES. Sex differences in stress responses: social rejection versus achievement stress. Biol Psychiatry. 2002;52:318-27.

3. Altemus M. Sex differences in depression and anxiety disorders: potential biological determinants. Horm Behav. 2006;50:534-8.

4. Nolen-Hoeksema S. Emotion regulation and psychopathology: the role of gender. Annu Rev Clin Psychol. 2012;8:161-87.

5. Gold PW, Chrousos GP. Organization of the stress system and its dysregulation in melancholic and atypical depression: high vs low $\mathrm{CRH} / \mathrm{NE}$ states. Mol Psychiatry. 2002;7:254-75.

6. Nater UM, Rohleder N, Gaab J, Berger S, Jud A, Kirschbaum C, et al. Human salivary alpha-amylase reactivity in a psychosocial stress paradigm. Int J Psychophysiol. 2005;55:333-42.

7. Ulrich-Lai YM, Herman JP. Neural regulation of endocrine and autonomic stress responses. Nat Rev Neurosci. 2009;10:397-409.

8. de Kloet ER, Joëls M, Holsboer F. Stress and the brain: from adaptation to disease. Nat Rev Neurosci. 2005;6:463-75.

9. Kajantie E, Phillips DIW. The effects of sex and hormonal status on the physiological response to acute psychosocial stress. Psychoneuroendocrinology. 2006;31:151-78.

10. Kudielka BM, Hellhammer J. Sex differences in endocrine and psychological responses to psychosocial stress in healthy elderly subjects and the impact of a 2week dehydroepiandrosterone treatment. J Clin Endocrinol Metab. 1998;83: 1756-61.

11. Gallucci WT, Baum A, Laue L, Rabin DS, Chrousos GP, Gold PW, et al. Sex differences in sensitivity of the hypothalamic-pituitary-adrenal axis. Health Psychol. 1993;12:420-5.

12. Kudielka BM, Buske-Kirschbaum A, Hellhammer DH, Kirschbaum C. HPA axis responses to laboratory psychosocial stress in healthy elderly adults, younger adults, and children: impact of age and gender. Psychoneuroendocrinology. 2004;29:83-98.

13. Kudielka BM, Kirschbaum C. Sex differences in HPA axis responses to stress: a review. Biol Psychol. 2005;69:113-32.

14. Carr AR, Scully A, Webb M, Felmingham KL. Gender differences in salivary alphaamylase and attentional bias towards negative facial expressions following acute stress induction. Cogn Emot. 2016;30:315-24.

15. Childs E, Dlugos A, De Wit H. Cardiovascular, hormonal, and emotional responses to the TSST in relation to sex and menstrual cycle phase. Psychophysiology. 2010;47:550-9.

16. Takai N, Yamaguchi M, Aragaki T, Eto K, Uchihashi K, Nishikawa Y. Gender-specific differences in salivary biomarker responses to acute psychological stress. Ann N Y Acad Sci. 2007;1098:510-5.

17. van Stegeren AH, Wolf OT, Kindt M. Salivary alpha amylase and cortisol responses to different stress tasks: impact of sex. Int J Psychophysiol. 2008;69:33-40.

18. Taylor SE, Klein LC, Lewis BP, Gruenewald TL, Gurung RA, Updegraff JA. Biobehavioral responses to stress in females: tend-and-befriend, not fight-or-flight. Psychol Rev. 2000;107:411-29.

19. Tamres LK, Janicki D, Helgeson VS. Sex differences in coping behavior: a metaanalytic review and an examination of relative coping. Pers Soc Psychol Rev. 2002;6:2-30.
20. Engert V, Vogel S, Efanov SI, Duchesne A, Corbo V, Ali N, et al. Investigation into the cross-correlation of salivary cortisol and alpha-amylase responses to psychological stress. Psychoneuroendocrinology. 2011;36:1294-302.

21. Andrews J, D'Aguiar C, Pruessner JC. The combined dexamethasone/TSST paradigm-a new method for psychoneuroendocrinology. PLoS ONE. 2012;7:e38994.

22. Andrews J, Pruessner JC. The combined propranolol/TSST paradigm-a new method for psychoneuroendocrinology. PLoS ONE. 2013;8:e57567.

23. Chrousos GP. Stress and disorders of the stress system. Nat Rev Endocrinol. 2009;5:374-81.

24. de Kloet ER, van der Vies J, de Wied D. The site of the suppressive action of dexamethasone on pituitary-adrenal activity. Endocrinology. 1974;94:61-73.

25. Winzer A, Ring C, Carroll D, Willemsen G, Drayson M, Kendall M. Secretory immunoglobulin $A$ and cardiovascular reactions to mental arithmetic, cold pressor, and exercise: effects of beta-adrenergic blockade. Psychophysiology. 1999;36:591-601.

26. Ali N, Pruessner JC. The salivary alpha amylase over cortisol ratio as a marker to assess dysregulations of the stress systems. Physiol Behav. 2012;106:65-72.

27. Vreeburg SA, Zitman FG, van Pelt J, Derijk RH, Verhagen JCM, van Dyck R, et al Salivary cortisol levels in persons with and without different anxiety disorders. Psychosom Med. 2010;72:340-7.

28. Bale TL, Epperson CN. Sex differences and stress across the lifespan. Nat Neurosci. 2015;18:1413-20.

29. Ali N, Nitschke JP, Cooperman C, Pruessner JC. Suppressing the endocrine and autonomic stress systems does not impact the emotional stress experience after psychosocial stress. Psychoneuroendocrinology. 2017;78:125-30.

30. Cohen, J. Statistical Power Analysis for the Behavioral Sciences-Second Edition Lawrence Erlbaum Associates Inc. Hillsdale, New Jersey, 1998;13.

31. Buchner A, Faul F, Erdfelder E. G*power. 1997. http://www.Psycho.Uniduesseldorf.De/aap/projects/gpower/how_to_use_gpower.html.

32. Kirschbaum C, Pirke K-M, Hellhammer DH. The 'Trier Social Stress Test'-a tool for investigating psychobiological stress responses in a laboratory setting. Neuropsychobiology. 1993;28:76-81.

33. Dressendörfer RA, Kirschbaum C, Rohde W, Stahl F, Strasburger CJ. Synthesis of a cortisol-biotin conjugate and evaluation as a tracer in an immunoassay for salivary cortisol measurement. J Steroid Biochem Mol Biol. 1992;43:683-92.

34. Gift AG. Visual analogue scales: measurement of subjective phenomena. Nurs Res. 1989;38:286-8.

35. Lorr M, McNair DM, Fisher SU. Evidence for bipolar mood states. J Pers Assess. 1982;46:432-6.

36. Gueorguieva R, Krystal JH. Move over ANOVA: progress in analyzing repeatedmeasures data and its reflection in papers published in the archives of general psychiatry. Arch Gen Psychiatry. 2004;61:310-7.

37. Barr DJ, Levy R, Scheepers C, Tily HJ. Random effects structure for confirmatory hypothesis testing: keep it maximal. J Mem Lang. 2013;68:255-78.

38. Pruessner JC, Kirschbaum C, Meinlschmid G, Hellhammer DH. Two formulas for computation of the area under the curve represent measures of total hormone concentration versus time-dependent change. Psychoneuroendocrinology 2003;28:916-31.

39. van Buuren S, Groothuis-Oudshoorn K. Mice: multivariate imputation by chained equations in R. J Stat Softw. 2011:45:1-67.

40. $\mathrm{R}$ Core Team. R: A language and environment for statistical computing. $\mathrm{R}$ Foundation for Statistical Computing, Vienna, Austria, 2013. http://www.Rproject.org/.

41. Bates D, Mächler M, Bolker B, Walker S. Fitting Linear Mixed-Effects Models Using Ime4. J Stat Softw. 2015;67:1-48. https://doi.org/10.18637/jss.v067.i01.

42. Preacher KJ, Curran PJ, Bauer DJ. Computational tools for probing interactions in multiple linear regression, multilevel modeling, and latent curve analysis. J Educ Behav Stat. 2006;31:437-48.

43. Miller R, Plessow F, Kirschbaum C, Stalder T. Classification criteria for distinguishing cortisol responders from nonresponders to psychosocial stress: evaluation of salivary cortisol pulse detection in panel designs. Psychosom Med. 2013;75:832-40.

44. Wilder J. Basimetric approach (law of initial value) to biological rhythms. Ann N Y Acad Sci. 1962;98:1211-20.

45. Young $E$, Korszun A. Women, stress, and depression: Sex differences in hypothalamic-pituitary-adrenal axis regulation. In Leibenluft, E (Ed.), Gender differences in mood and anxiety disorders: From bench to bedside (pp. 31-52). Washington, DC: American Psychiatric Press. 1999.

46. Heck AL, Handa RJ. Sex differences in the hypothalamic-pituitary-adrenal axis' response to stress: an important role for gonadal hormones. Neuropsychopharmacology. 2019;44:45-58.

47. Douma SL, Husband C, O'Donnell ME, Barwin BN, Woodend AK. Estrogen-related mood disorders: reproductive life cycle factors. ANS Adv Nurs Sci. 2005;28:364-75. 
1680

48. Troisi A. Gender differences in vulnerability to social stress: a Darwinian perspective. Physiol Behav. 2001;73:443-9.

49. Galea LA, McEwen BS, Tanapat P, Deak T, Spencer RL, Dhabhar FS. Sex differences in dendritic atrophy of CA3 pyramidal neurons in response to chronic restraint stress. Neuroscience. 1997;81:689-97.

50. Spear LP. Heightened stress responsivity and emotional reactivity during pubertal maturation: Implications for psychopathology. Dev Psychopathol. 2009;21:87-97.

51. Lazarus RS, Folkman S. Coping and Adaptation. In Gentry, WD (Ed.), The Handbook of Behavioral Medicine (pp. 282-325). New York: Guilford. 1984.

52. Het $\mathrm{S}$, Wolf OT. Mood changes in response to psychosocial stress in healthy young women: effects of pretreatment with cortisol. Behav Neurosci 2007;121:11-20.

53. Het S, Schoofs D, Rohleder N, Wolf OT. Stress-induced cortisol level elevations are associated with reduced negative affect after stress: indications for a moodbuffering cortisol effect. Psychosom Med. 2012;74:23-32.
54. Luecken LJ, Appelhans BM. Early parental loss and salivary cortisol in young adulthood: the moderating role of family environment. Dev Psychopathol. 2006;18:295-308.

55. Ehlert U, Gaab J, Heinrichs M. Psychoneuroendocrinological contributions to the etiology of depression, posttraumatic stress disorder, and stress-related bodily disorders: the role of the hypothalamus-pituitary-adrenal axis. Biol Psychol. 2001;57:141-52.

56. Tuross N, Patrick RL. Effects of propranolol on catecholamine synthesis and uptake in the central nervous system of the rat. J Pharm Exp Ther. 1986;237:739-45.

57. Andreano JM, Arjomandi H, Cahill L. Menstrual cycle modulation of the relationship between cortisol and long-term memory. Psychoneuroendocrinology. 2008;33:874-82. 